\title{
Étude de la sensibilité à la fissuration à chaud d'un alliage CuCrZr au cours du soudage par faisceau d'électrons
}

\author{
J.-M. Drezet ${ }^{1}$, D. Ayrault², J. Wisniewski², M. Barras², P. Pilvin³, \\ D. Carron ${ }^{3}$ et F. Primaux ${ }^{4}$
}

1 Laboratoire de Simulation des Matériaux (LSMX), École Polytechnique Fédérale de Lausanne, MXG 338, Ecublens, Lausanne, 1015, Suisse

e-mail : jean-marie.drezet@epfl.ch

2 CEA, DEN, DM2S, SEMT, LTA, 91191 Gif-sur-Yvette, France

e-mail : danielle.ayrault@cea.fr ; jean.wisniewski@cea.fr; michel.barras@cea.fr

3 Laboratoire d'Ingénierie des Matériaux de Bretagne (LIMatB), Université de Bretagne-Sud/UEB, Centre de Recherche, rue de Saint-Maudé, BP 92116, 56321 Lorient Cedex, France e-mail : philippe.pilvin@univ-ubs.fr; demis.carron@univ-ubs.fr

4 Le Bronze Industriel, Z.I. Voie de Châlons, RD 977, 51600 Suippes, France e-mail : fprimaux@lebronzeindustriel.com

Mots-clés :

Alliage $\mathrm{CuCrZr}$; caractérisation thermo-physique et thermo-mécanique ; fissuration à chaud; soudage par faisceau d'électrons; essais de fissuration JWRI ; analyse numérique
Résumé - L'alliage CuCrZr est utilisé dans les machines fusion notamment pour des composants refroidis face au plasma, à cause de ses bonnes propriétés thermiques et mécaniques. Cependant, le retour d'expérience sur plusieurs tokamaks, et plus particulièrement Tore Supra en France, a montré que cet alliage est très sensible au phénomène de fissuration à chaud au cours de son assemblage par faisceau d'électrons avec apparition de fissures de solidification dans la zone pâteuse. Afin de caractériser la sensibilité de l'alliage à la fissuration à chaud, des essais de fissuration basés sur l'essai JWRI (Joining and Welding Research Institute) ont été menés. Une ligne de fusion par faisceau d'électrons est réalisée sur une éprouvette parallélépipédique de faible épaisseur équipée avec des thermocouples et maintenue à une de ses extrémités. Selon la largeur de l'éprouvette, l'essai est fissurant on non fissurant. En effet, lorsque la largeur de l'éprouvette diminue, les conditions thermo-mécaniques favorables à l'apparition d'une fissure peuvent apparaître. Les essais JWRI ont ensuite été analysés à l'aide de la simulation numérique et différents critères d'amorçage de la fissuration à chaud ont été appliqués. Les propriétés thermo-physiques et thermo-mécaniques manquantes de l'alliage CuCrZr et nécessaires pour la mise en place des modèles ont été déterminées en associant des essais en laboratoire et une analyse numérique. La déformation et la vitesse de déformation viscoplastiques considérées comme indicateurs thermo-mécaniques au regard de la fissuration à chaud ont été calculées. Les conditions d'amorçage de la fissuration à chaud sont ensuite comparées aux conditions de propagation d'une fissure en utilisant une approche numérique simple.
Key words:

CuCrZr alloy; thermophysical and thermomechanical characterization; hot tearing; electron beam welding; JWRI cracking tests; numerical analysis

\begin{abstract}
Hot tearing susceptibility of a CuCrZr alloy during electron beam welding. The precipitation hardened $\mathrm{CuCrZr}$ alloy is used in fusion machines for the heat sink of intensely cooled plasma facing components due to its good thermal and mechanical properties. Albeit, the feedback from its application in Tore Supra (French Tokamak) showed that this alloy is very sensitive to hot tearing during electron beam welding. The solidification cracks occur in solidifying parts undergoing tensile stresses transmitted to the mushy zone by the coherent solid underneath. In order to characterize the hot tearing susceptibility of the CuCrZr alloy, welding tests based on the investigations of the Joining and Welding Research Institute (JWRI) have been performed. Electron beam fusion lines are realized on a thin parallelepipedic plate equipped with thermocouples and clamped at its extremity. As the width of the plate decreases, conditions leading to the formation of hot tears appear in the run-in, thus definig a critical width hot tearing wise. The JWRI welding tests are then analysed by means of numerical modelling and available hot tearing criteria. Missing thermophysical and thermomechanical properties of the CuCrZr alloy are determined by associating laboratory tests and numerical analysis. The viscoplastic strain and viscoplastic strain rate undergone by the solidifying alloy are considered as hot tearing indicators. The indicators values at the onset of the hot tearing are determined for this alloy. In addition, HT initiation conditions are compared with propagation conditions using a simple numerical approach.
\end{abstract}


alliage $\mathrm{CuCrZr}$, durci par précipitation, est utilisé comme matériau de structure dans la fabrication des composants internes du réacteur expérimental de fusion contrôlée Tore Supra implanté au CEA/Cadarache et dans d'autres réacteurs de fusion tels que le JET en Grande-Bretagne [1]. Il est également candidat pour des composants de la future machine ITER [2]. Cet alliage a été initialement sélectionné pour ses bonnes propriétés thermo-mécaniques à température élevée et ses propriétés thermo-physiques. Le retour d'expérience, notamment de Tore Supra et du JET, concernant le soudage homogène par faisceau d'électrons du CuCrZr, indique toutefois des problèmes récurrents de fissuration à chaud [3]. Ce type de défaut de fissuration à chaud apparaît dans la zone pâteuse en fin de solidification pour des fractions volumiques solides élevées [4-6]. Les fissures à chaud de solidification résultent de l'effet combiné d'une mauvaise alimentation en liquide dans les espaces interdendritiques et d'importantes déformations en tension, dues au retrait du matériau macroscopiquement solidifié, qui ne peuvent être supportées par la zone pâteuse.

Dans ce contexte, on souhaite caractériser la sensibilité à la fissuration à chaud de l'alliage $\mathrm{CuCrZr}$. Le but de cette étude est d'évaluer les conditions thermo-mécaniques conduisant à l'amorçage d'une fissure à chaud au cours du soudage d'un alliage $\mathrm{Cu}$ $\mathrm{CrZr}$. On utilise, pour ce faire, une approche couplant expérimentation et simulation numérique.

Il existe de nombreux essais de fissuration à chaud qui consistent, pour la majorité d'entre eux, en la réalisation d'une ligne de fusion sur une éprouvette de faible épaisseur afin de générer plus facilement une fissure [7-11]. La géométrie de l'éprouvette est différente selon les sources. Une éprouvette parallélépipédique est utilisée, par exemple, dans $[7,8]$ et une ligne de fusion centrale et droite [7] ou oblique [8] est alors effectuée. Inspirées par l'essai Houldcroft [9], des éprouvettes de géométrie trapézoïdale sont aussi utilisées [10,11]. La ligne de fusion est réalisée depuis la partie la plus étroite de l'éprouvette qui favorise l'apparition d'une fissure vers la partie la plus large, pour laquelle les conditions d'amorçage ne sont plus présentes [11]. Parmi ces différents tests, nous avons retenu pour notre étude les essais JWRI (Joining and Welding Research Institute) inspirés par les travaux de
Shibahara et al. [7]. Les essais menés ont ensuite été analysés à l'aide de la simulation numérique.

De nombreux critères de fissuration à chaud apparaissent dans la littérature [12], deux d'entre eux sont appliqués ici. D'une part, on s'intéresse au critère phénoménologique RDG $[4,13]$ qui, à partir du calcul de la composante d'ouverture de la vitesse de déformation viscoplastique, pour une fraction volumique solide de $95 \%$, correspondant à la température de coalescence de l'alliage, permet d'évaluer la chute de pression interdendritique. Plus celle-ci est élevée, plus le risque de fissuration à chaud est grand. Cette approche est applicable lorsque les films liquides sont continus et que les grains n'ont pas encore totalement coalescé, ce qui correspond à une fraction volumique solide de l'ordre de $95 \%$ [6]. L'intervalle de température, au sein duquel existe un film liquide continu, est le BTR (Brittle Temperature Range) [14,15]. La borne supérieure du BTR correspond à la température en dessous de laquelle les dendrites forment un réseau incohérent avec un film liquide continu entre elles mais où l'alimentation en liquide est quasiment impossible due à la très faible perméabilité du milieu. La borne inférieure correspond, quant à elle, à la température pour laquelle il n'y a plus aucune ductilité car des ponts solides commencent à se former entre les dendrites (coalescence), faisant croître la ductilité du milieu qui peut alors supporter les chargements en tension [16]. Le minimum de la courbe de ductilité en « $U$ » est un paramètre crucial car il limite la déformation que peut supporter la zone pâteuse sans créer une fissure. Par exemple, un minimum de ductilité entre $0,5 \%$ et $1 \%$ à $1410{ }^{\circ} \mathrm{C}$ a été calculé pour un acier austénitique AISI 321 à l'aide d'essais Varestraint et JWRI par Kerrouault [17]. Cerri [18], quant à lui, a identifié une valeur de $1 \%$ pour deux aciers différents. Aucune donnée n'existe pour les alliages de cuivre.

Dans cette étude, nous allons également nous intéresser au critère de Kerrouault [17] qui considère la composante transverse de la déformation viscoplastique comme indicateur de la sensibilité à la fissuration à chaud, dans le domaine BTR.

Pour la présente étude, des essais de fissuration à chaud sont menés et analysés à l'aide de la simulation numérique. En se basant sur les deux critères retenus, des indicateurs thermo-mécaniques d'amorçage de la fissuration à chaud à l'arrière du bain de 


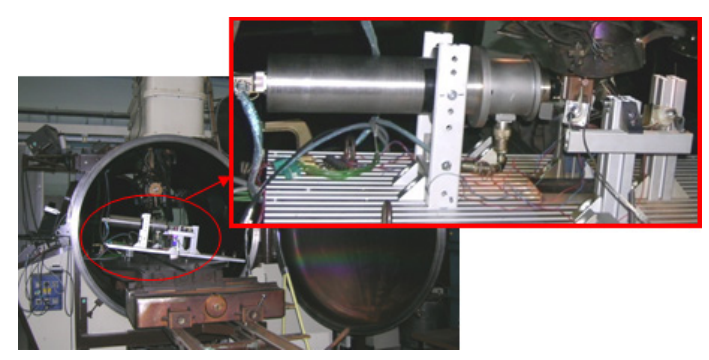

(a)
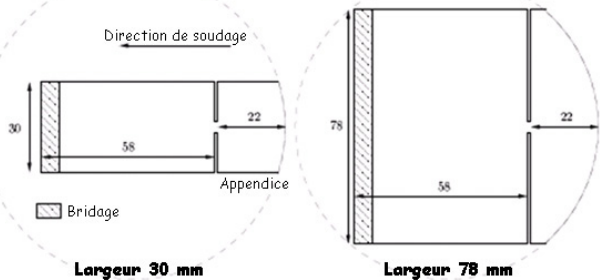

(b)

Fig. 1. Essais JWRI. Installation de soudage par faisceau d'électrons et montage instrumental (a) Eprouvettes JWRI de largeur variable (b) [19].

Fig. 1. JWRI tests. Electron beam welding apparatus and experimental device (a) JWRI specimen with variable width (b) [19].

fusion sont évalués. Enfin, à l'aide du modèle numérique les conditions d'amorçage du défaut sont comparées aux conditions de propagation.

\section{Essais instrumentés JWRI}

Le matériau étudié est un alliage $\mathrm{CuCr} 1 \mathrm{Zr}$ selon la norme DIN 17672, de composition chimique entre $0,5 \%$ et $1,2 \%$ pour le chrome et entre $0,03 \%$ et $0,3 \%$ pour le zirconium en poids pour-cent [19]. Il a été élaboré par la société «Le Bronze Industriel » sous forme $\mathrm{d}$ 'un rondin de diamètre $100 \mathrm{~mm}$ selon une gamme de fabrication comportant une mise en solution à $1000^{\circ} \mathrm{C}$ pendant $2 \mathrm{~h}$, une étape de filage à chaud suivie d'une trempe à l'eau. Un traitement thermique de revenu à $480^{\circ} \mathrm{C}$ pendant $7 \mathrm{~h}$ suivi d'un refroidissement à l'air confère à l'alliage ses propriétés mécaniques finales.

Les essais de type JWRI ont été réalisés au laboratoire LTA du CEA [19]. Des éprouvettes parallélépipédiques de faible épaisseur 2,5 mm ont été prélevées dans un plan normal à l'axe du rondin. Une des extrémités de l'éprouvette présente un appendice où s'établit la montée en puissance du faisceau. Le ligament entre les gorges présente la même largeur que celle du bain de soudage. L'autre extrémité de l'éprouvette quant à elle est maintenue dans un système de bridage. L'essai consiste à réaliser une ligne de fusion par faisceau d'électrons depuis l'appendice, qui chute au passage du faisceau entre les gorges, vers l'extrémité bridée. La faible épaisseur et les conditions opératoires sont telles que les largeurs endroit et envers de la zone fondue sont identiques, c'est-à-dire que le champ de température est supposé peu variable dans l'épaisseur.
Les éprouvettes sont instrumentées à l'aide de thermocouples et un système de vision (caméra et objectif), positionné dans un caisson étanche sous pression atmosphérique dans l'enceinte sous vide, permet de filmer la scène de soudage (Fig. 1a). La configuration de soudage et les paramètres opératoires sont maintenus constants pour tous les essais : vitesse de soudage de $50 \mathrm{~cm} \mathrm{~min}^{-1}$, tension de $50 \mathrm{kV}$, intensité de $70 \mathrm{~mA}$; la puissance fournie par le faisceau est donc de $3,5 \mathrm{~kW}$. La longueur soudée est de l'ordre de $60 \mathrm{~mm}$; la durée de soudage est d'environ $7 \mathrm{~s}$. Par ailleurs, le point de focalisation du faisceau se situe $20 \mathrm{~mm}$ au dessus de la face supérieure de l'éprouvette. Seule la largeur de l'éprouvette varie selon les essais (Fig. 1b), permettant ainsi d'obtenir des cas fissurants ou non fissurants.

Des essais JWRI ont ainsi été réalisés avec des largeurs d'éprouvettes variables et répétés pour certains d'entre eux, afin de valider la reproductibilité des observations (Tab. 1). On constate que les essais en largeur $30 \mathrm{~mm}$ et $40 \mathrm{~mm}$ sont toujours fissurants (3 fois). Les trois essais en largeur $78 \mathrm{~mm}$ sont au contraire non fissurants (Fig. 2) ainsi que l'essai de largeur $60 \mathrm{~mm}$.

Concernant la largeur $50 \mathrm{~mm}$, les observations indiquent que l'on se situe probablement près du seuil critique de largeur d'éprouvette en dessous duquel on observe la fissuration.

Des mesures en cours de soudage, à l'aide de la caméra, de la largeur et de la longueur de bain ont été réalisées; les résultats pour un essai fissurant et un essai non fissurant sont donnés tableau 2 et figure 3. On observe une largeur de cordon très similaire dans les deux cas, alors que la longueur de bain est quant à elle légèrement supérieure dans le cas fissurant. Pour les deux essais, 

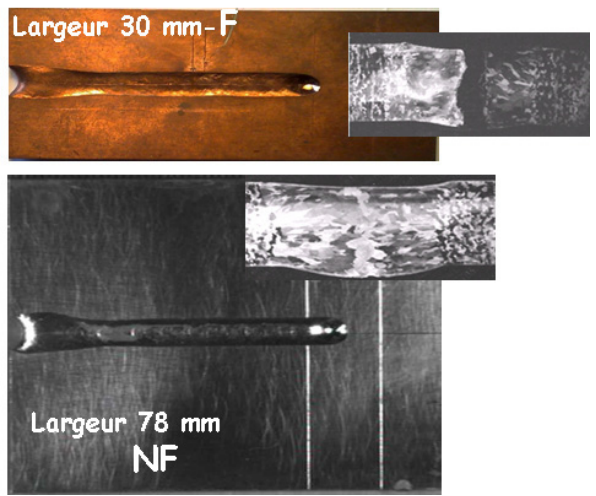

Fig. 2. Exemple d'essais JWRI fissurant (largeur éprouvette $30 \mathrm{~mm}$ ) et non fissurant (largeur éprouvette $78 \mathrm{~mm}$ ) [19].

Fig. 2. Examples of JWRI cracking test (specimen width $30 \mathrm{~mm}$ ) and no cracking test (specimen width $78 \mathrm{~mm}$ ) [19].

Tableau 1. Synthèse des essais JWRI [19] (NF : non fissurant, $\mathrm{F}$ : fissurant).

Table 1. JWRI tests synthesis [19] (NF: no cracking, F: cracking).

\begin{tabular}{ccc}
\hline Largeur $(\mathrm{mm})$ & $\mathrm{F}$ & $\mathrm{NF}$ \\
\hline 30 & $3 \times$ & \\
40 & $\times$ & \\
50 & $2 \times$ & $2 \times$ \\
60 & & $1 \times$ \\
78 & & $3 \times$ \\
\hline
\end{tabular}

Tableau 2. Dimensions du bain de fusion lors du soudage d'éprouvettes JWRI [19] (NF : non fissurant, $\mathrm{F}$ : fissurant).

Table 2. Welding pool dimensions during JWRI tests [1] (NF: no cracking, F: cracking).

\begin{tabular}{cccc}
\hline Largeur éprouvette (mm) & Type d'essai & \multicolumn{2}{c}{ Taille du bain $(\mathrm{mm})$} \\
& & Largeur & Longueur \\
\hline 30 & $\mathrm{~F}$ & 4,7 & 6 \\
78 & $\mathrm{NF}$ & 4,5 & 5,3 \\
\hline
\end{tabular}

on observe par ailleurs un régime transitoire après chute de l'appendice sur une longueur de l'ordre de $10 \mathrm{~mm}$ où la largeur du bain s'accroit (Fig. 2).

Ces essais, outre l'avantage de fournir des données expérimentales, seront simulés afin de calculer les indicateurs thermomécaniques permettant de les discriminer au regard de la fissuration à chaud. Les propriétés thermo-physiques et thermomécaniques du matériau sont cependant nécessaires pour mener les simulations numériques.

\section{Caractérisations du matériau}

\subsection{Propriétés thermo-physiques}

Pour effectuer l'analyse par éléments finis, il est nécessaire de disposer, dans un domaine de température depuis l'ambiante jusqu'à l'état liquide, d'une part, de la loi de comportement thermo-mécanique et, d'autre part, des propriétés thermo-physiques de l'alliage. Concernant ces dernières, les données manquantes ont été déterminées en associant des essais en laboratoire à l'École Polytechnique Fédérale de Lausanne (EPFL) et une analyse numérique [20]. La figure 4a présente le chemin de solidification de l'alliage. Le modèle retenu pour ce dernier est basé sur le modèle de Scheil-Gulliver car les vitesses de refroidissement sont très élevées en soudage. Les températures de solidus et liquidus sont respectivement de $1048{ }^{\circ} \mathrm{C}$ et $1080{ }^{\circ} \mathrm{C}$; cet alliage présente donc un très faible intervalle de solidification de $32{ }^{\circ} \mathrm{C}$. La chaleur latente de fusion est, quant à elle, prise égale à celle du cuivre pur $\left(204 \mathrm{~kJ} \mathrm{~kg}^{-1}\right)$ due à la faible teneur en éléments d'alliage.

La conductivité thermique a été identifiée par méthode inverse à l'aide d'essais de type coulée 1D instrumentés avec des thermocouples [20]. Sur la figure $4 \mathrm{~b}$ sont données les évolutions avec la température de la conductivité thermique $\lambda$ et la chaleur spécifique $c_{p}$ pour l'alliage $\mathrm{CuCr} 1 \mathrm{Zr}$ et pour le cuivre pur pour comparaison. La valeur élevée de la conductivité thermique pour une température supérieure au liquidus est due aux mouvements de convection dans le liquide.

\subsection{Loi de comportement mécanique}

Le comportement thermo-mécanique de l'alliage $\mathrm{CuCr} 1 \mathrm{Zr}$ a été caractérisé à l'aide de tests dilatométriques et d'essais de traction isothermes à différentes vitesses de déformation sur une machine Gleeble 3500 à l'Université de Bretagne-Sud (UBS) et pour des températures allant de $20^{\circ} \mathrm{C}$ à $1000^{\circ} \mathrm{C}$ [21]. Certains essais à $20^{\circ} \mathrm{C}$ ont été menés sur une machine Instron 30.

Un modèle élasto-visco-plastique (EVP) à cinq coefficients (écrouissage isotrope) a été identifié avec l'aide du logiciel SiDoLo [22].

La loi utilisée inclut les hypothèses suivantes:

- le matériau est considéré isotrope ;

- les effets de restauration sont négligés;

- il y a partition des déformations : la déformation totale est la somme des trois composantes thermique, élastique et viscoplastique. 


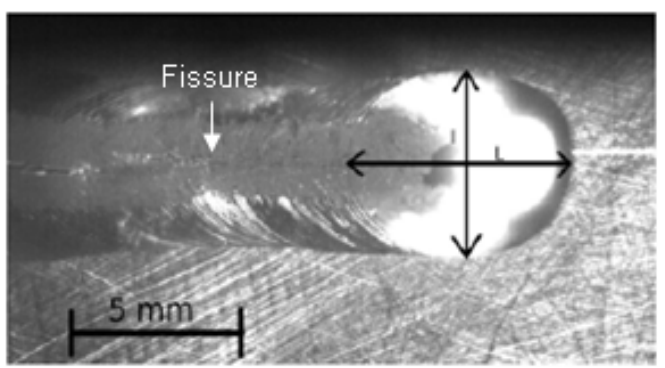

(a)

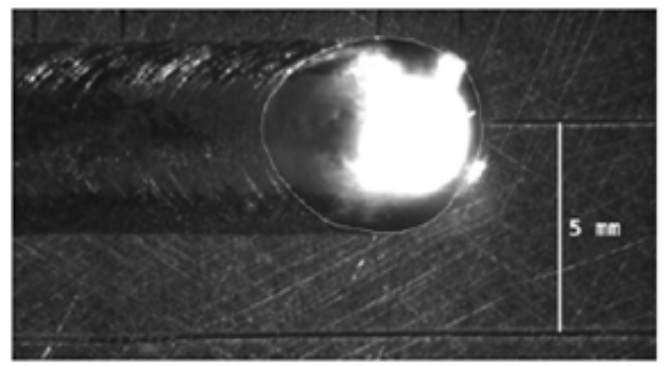

(b)

Fig. 3. Vues de la scène de soudage d'essais JWRI (a) cas fissurant (largeur éprouvette $30 \mathrm{~mm}$ ) (b) ; cas non fissurant (largeur éprouvette $78 \mathrm{~mm}$ ).

Fig. 3. JWRI tests welding scene. (a) Cracking test (specimen width $30 \mathrm{~mm}$ ); (b) no cracking test (specimen width $78 \mathrm{~mm}$ ).

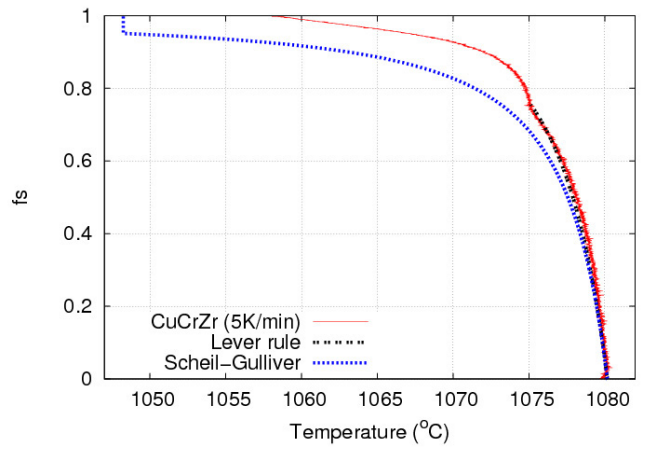

(a)

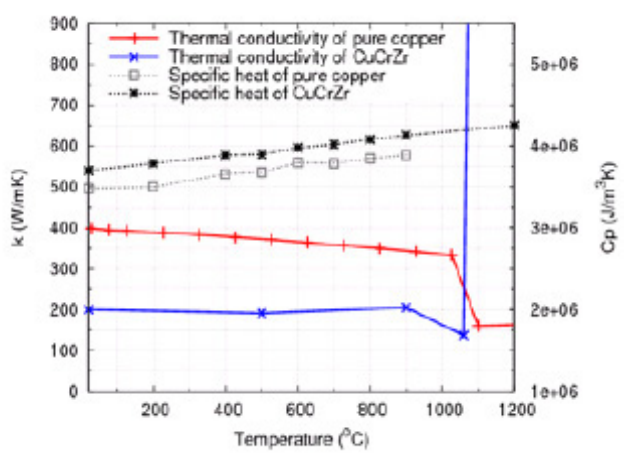

(b)

Fig. 4. Propriétés thermo-physiques de l'alliage CuCr1Zr [20]. Chemin de solidification (a) Conductivité thermique et chaleur spécifique (b).

Fig. 4. Thermophysical properties of $\mathrm{CuCr1Zr}$ alloy [20]. Solidification path (a) - Thermal conductivity and specific heat (b).

Le critère de plasticité de type Von Mises avec écrouissage isotrope est retenu. Une loi de Voce pour l'écrouissage est utilisée.

D'après ce modèle, la vitesse de déformation viscoplastique s'écrit :

$$
\dot{\varepsilon}^{\mathrm{vp}}=\left[\frac{\sigma_{\mathrm{eq}}-\sigma_{y}-Q\left(1-\mathrm{e}^{k \varepsilon}\right)}{K}\right]^{n}
$$

où $K$ est la consistance viscoplastique, $\varepsilon$ la déformation viscoplastique accumulée, $\sigma_{\mathrm{eq}}$ la contrainte équivalente de Von Mises, $\sigma_{y}$ la limite d'élasticité, $n$ un exposant, $Q$ et $b$ des paramètres qui décrivent l'écrouissage.

Une comparaison calcul/expérience est donnée sur la figure 5 pour des températures moyennes entre 600 et $800^{\circ} \mathrm{C}$ et élevées entre 850 et $1000^{\circ} \mathrm{C}$ [21].

\section{Analyse numérique}

Des calculs éléments finis (2D contraintes planes) ont été menés à l'aide du code de calcul Abaqus 6.8 [23]. L'hypothèse 2D est justifiée par la géométrie du cordon. En effet, les macrographies montrent que la zone fondue pénètre complètement dans l'épaisseur de l'éprouvette et que ses bords sont pratiquement orthogonaux au plan de la plaque (Fig. 2). Ainsi, le champ de température, qui varie très peu dans l'épaisseur, est estimé par un modèle 2D de Rosenthal valide pour les plaques minces [24] :

$$
T(x, y)=T_{0}+\frac{\beta P}{2 k s} \sqrt{\frac{\alpha}{\pi V r}} \mathrm{e}^{\frac{-V(x+r)}{2 \alpha}}
$$

et

$$
r^{2}=x^{2}+y^{2}
$$

où $k$ est la conductivité thermique de l'alliage $\mathrm{CuCr} 1 \mathrm{Zr}, V$ la vitesse de soudage égale 


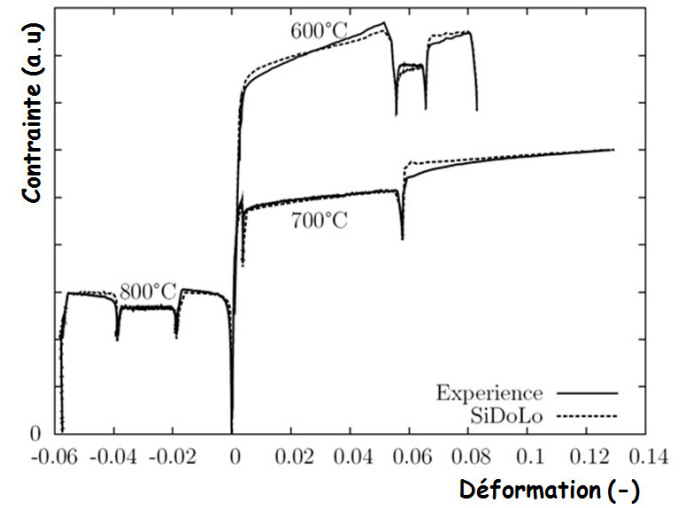

(a)

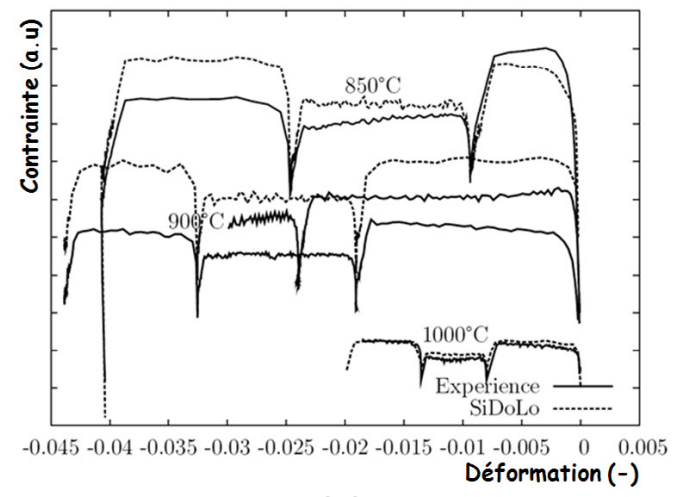

(b)

Fig. 5. Propriétés thermo-mécaniques de l'alliage $\mathrm{CuCr} 1 \mathrm{Zr}$ [21]. Températures de $600{ }^{\circ} \mathrm{C}$ à $800{ }^{\circ} \mathrm{C}$ (a) - Températures de $850^{\circ} \mathrm{C}$ à $1000{ }^{\circ} \mathrm{C}(\mathrm{b})$.

Fig. 5. Thermomechanical properties of $\mathrm{CuCr} 1 \mathrm{Zr}$ alloy [21]. Temperatures from $600{ }^{\circ} \mathrm{C}$ to $800{ }^{\circ} \mathrm{C}$ (a) Temperatures from $850{ }^{\circ} \mathrm{C}$ to $1000^{\circ} \mathrm{C}(\mathrm{b})$.

à $50 \mathrm{~cm} \mathrm{~min}^{-1}, \alpha$ la diffusivité thermique de $34,75 \mathrm{~mm}^{2} \mathrm{~s}^{-1}$, s l'épaisseur de la plaque égale à $2,5 \mathrm{~mm}, P$ la puissance nominale de $3,5 \mathrm{~kW}$ et $\beta$ le coefficient d'absorption.

Le champ thermique ainsi calculé dans le solide est très représentatif des températures expérimentales obtenues en certains points de l'éprouvette à l'aide de thermocouples au cours des essais. Le coefficient d'absorption $\beta$ est ajusté à $83 \%$ de telle sorte que la taille du bain fondu soit proche de celle estimée expérimentalement.

La composante $G_{x}$, selon l'axe $x$ de soudage, du gradient thermique à la température de solidus est un paramètre important pour l'amorçage du défaut de fissuration à chaud [6]. Le calcul analytique de $G_{x}$ à partir de l'équation (2) donne $124 \mathrm{~K} \mathrm{~mm}^{-1}$. La vitesse de refroidissement à la température de solidus donnée par la quantité « $-G_{x} \cdot V$ » est donc égale à $-1033 \mathrm{~K} \mathrm{~s}^{-1}$. Ces deux quantités restent constantes pour tous les essais car la vitesse de soudage et la puissance du faisceau ne varient pas. Les calculs thermomécaniques sont découplés; on suppose que la mécanique $\mathrm{n}^{\prime}$ influe pas sur la thermique, avant l'apparition d'une fissure. Le champ de température dans l'éprouvette est calculé à l'aide de l'équation (2) en déplaçant le faisceau le long de l'axe $x$ d'une quantité « $V \mathrm{~d} t$ ». Le champ obtenu (Fig. 6b) est ensuite utilisé comme chargement thermique pour le calcul mécanique.

Seule la moitié de l'éprouvette est modélisée, compte tenu de la symétrie du problème. Le maillage est particulièrement fin dans la zone centrale de l'éprouvette concernée par la fissuration à chaud. Ce phénomène intervient en effet dans la zone pâteuse à l'arrière du bain et pour un intervalle de solidification particulièrement étroit. Un maillage raffiné avec des éléments d'une taille de $200 \times 200$ micromètres est utilisé (Fig. 6a). Les conditions aux limites mécaniques consistent en un blocage de tous les nœuds à l'extrémité de l'éprouvette bridée et d'une condition de symétrie sur l'axe X.

Pour traiter l'état liquide du bain de fusion dans Abaqus, les composantes de la déformation plastique devant être remises à zéro, nous utilisons la technique « d'activation/réactivation » [25]. Les éléments de maillage qui atteignent la température de liquidus de $1080^{\circ} \mathrm{C}$ sont enlevés du calcul; ils sont réintroduits sans déformation plastique quand leur température devient inférieure à $1080^{\circ} \mathrm{C}$.

Les deux indicateurs thermomécaniques de fissuration à chaud décrits dans l'introduction sont calculés pour la configuration de l'étude. D'une part, la composante transverse de la déformation viscoplastique $\varepsilon^{\mathrm{vp}}$ perpendiculaire au gradient thermique, donc la composante yy dans le repère macroscopique, est calculée dans le domaine BTR [17]. Dans la littérature, on considère que le BTR se situe pour des fractions volumiques solides entre $70 \%$ [17] et $95 \%$ [14], ce qui correspond d'après la figure $4 \mathrm{a}$ à des températures respectives de $1075{ }^{\circ} \mathrm{C}$ à $1050{ }^{\circ} \mathrm{C}$ (température de coalescence).

Le second indicateur basé sur le critère RDG $[4,13]$ consiste en la composante transverse de la vitesse de 


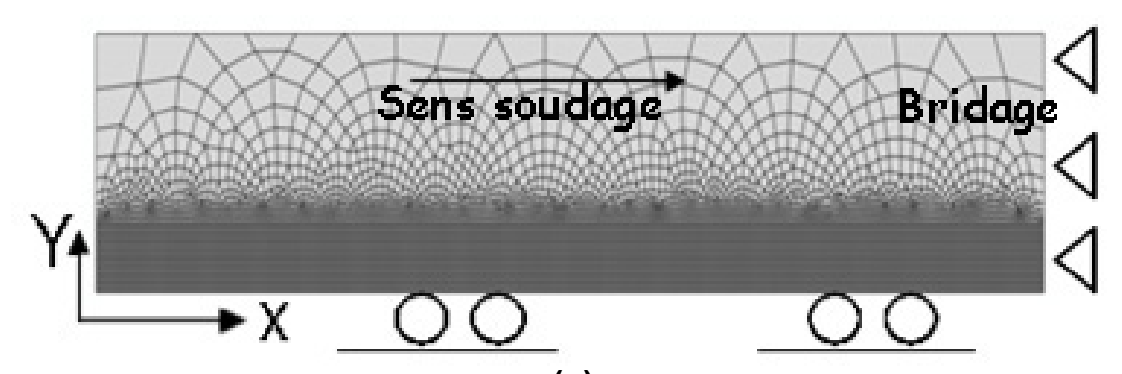

(a)

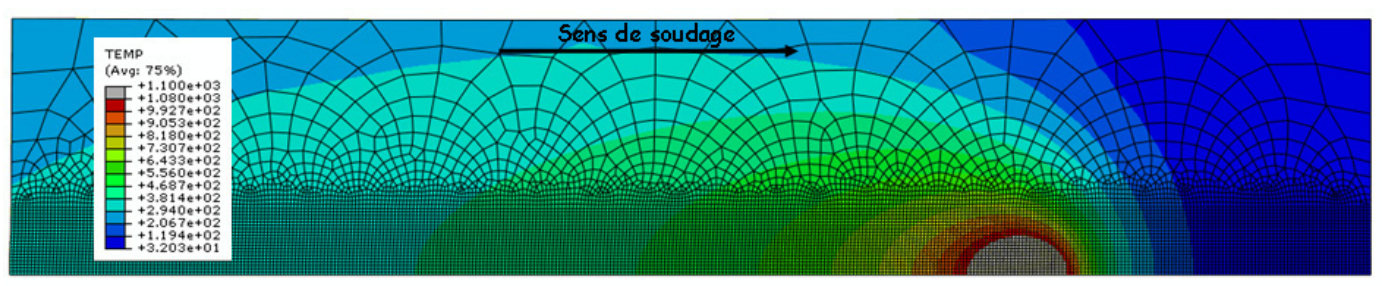

(b)

Fig. 6. Maillage de l'éprouvette et conditions aux limites mécaniques (a). Champ de température (b).

Fig. 6. Specimen mesh and mechanical boundary conditions (a). Temperature field (b).

déformation viscoplastique $\dot{\varepsilon}^{\mathrm{vp}}$ permettant ensuite d'évaluer la chute de pression interdendritique de cavitation. Le calcul se fait à la température de coalescence donc pour une fraction solide de l'ordre de $95 \%$.

Plus ces indicateurs sont élevés, plus le risque de fissuration à chaud est grand. Ils sont évalués uniquement au refroidissement et le long de l'axe de soudage car la fissure centrale s'amorce uniquement à cet endroit pour ce type de configuration d'éprouvette.

\section{Analyse des résultats}

Sont présentés ici les résultats des calculs sur éprouvettes JWRI fissurées et non fissurées. Tout d'abord, on analyse la valeur des indicateurs au tout début de la ligne de fusion pour différentes largeurs d'éprouvettes puisque la fissure, pour une configuration fissurante, est initiée dans cette zone. Il est ainsi possible de cerner les ordres de grandeurs des conditions thermo-mécaniques d'amorçage de la fissuration à chaud. On étudie ensuite les conditions de propagation d'une fissure.

\subsection{Conditions d'amorçage de la fissuration à chaud}

L'évolution des deux indicateurs de la fissuration à chaud le long de l'axe de la ligne de fusion est donnée sur la figure 7 pour différentes largeurs d'éprouvettes JWRI, les autres paramètres de soudage restant les mêmes [23].

Pour une largeur donnée, les indicateurs présentent un maximum au tout début de la ligne de fusion, où s'initient les fissures, et diminuent ensuite très rapidement sur une longueur d'environ $10 \mathrm{~mm}$, correspondant à une zone transitoire après chute de l'appendice, pour se stabiliser à de faibles valeurs dans la partie de l'éprouvette qui voit un régime établi de soudage. Ceci explique pourquoi les fissures sont amorcées dans le domaine transitoire. On a noté expérimentalement une augmentation de la largeur du bain dans cette zone. Des calculs prenant en compte ce phénomène (facteur $\beta$ dans Éq. (2) à $90 \%$ sur les 10 premiers $\mathrm{mm}$ ) indiquent une très faible influence sur les indicateurs.

On observe une diminution de la déformation viscoplastique d'ouverture de $4,2 \times$ $10^{-3}$ en largeur $78 \mathrm{~mm}$ (configuration non fissurante) jusqu'à $1,7 \times 10^{-3}$ pour la configuration fissurante en largeur $30 \mathrm{~mm}$ (Fig. 7a). Les mêmes tendances sont observées pour la vitesse de déformation viscoplastique transverse qui varie depuis une valeur de $0,22 \mathrm{~s}^{-1}$ en largeur $78 \mathrm{~mm}$ jusqu'à $0,10 \mathrm{~s}^{-1}$ en largeur $30 \mathrm{~mm}$ (Fig. 7b).

Pour la largeur critique d'éprouvette estimée expérimentalement autour de $50 \mathrm{~mm}$, les valeurs des indicateurs sont donc de $2,7 \times 10^{-3}$ pour la déformation viscoplastique d'ouverture et de $0,18 \mathrm{~s}^{-1}$ pour la vitesse de 


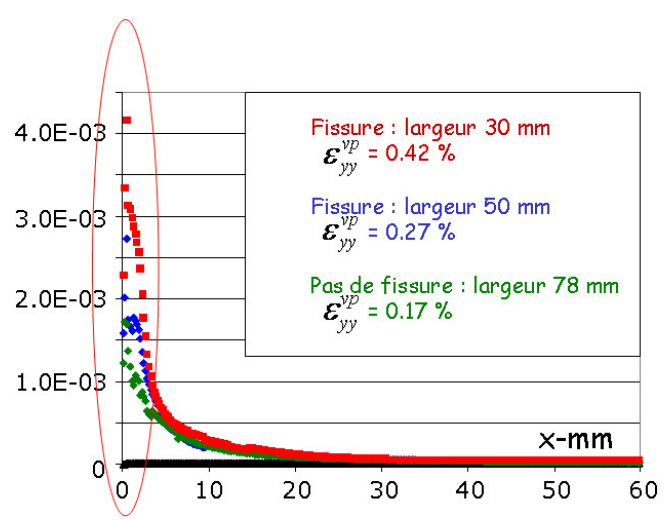

(a)

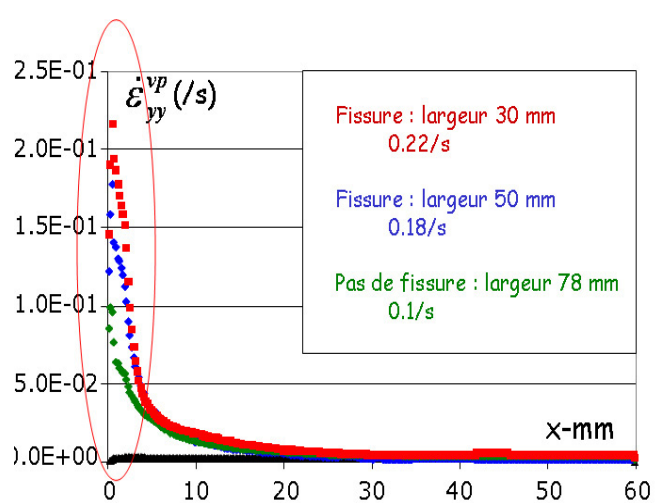

(b)

Fig. 7. Déformation viscoplastique (a) et vitesse de déformation viscoplastique (b) à la température de coalescence pour trois largeurs d'éprouvette $30 \mathrm{~mm}$ fissurée, $50 \mathrm{~mm}$ fissurée et $78 \mathrm{~mm}$ non fissurée en fonction de la longueur de l'éprouvette (selon l'axe de soudage $x$ ) (cf. Fig. 4) [23].

Fig. 7. Viscoplastic strain (a) and viscoplastic strain rate (b) at the coalescence temperature for three specimen widths $30 \mathrm{~mm}$ cracked, $50 \mathrm{~mm}$ cracked and $78 \mathrm{~mm}$ uncracked as a function of specimen length (along welding axis $x$ ) (see Fig. 4) [23].

déformation viscoplastique. La valeur de déformation de $2,7 \times 10^{-3}$ indique que l'alliage présente très peu de ductilité dans le BTR par rapport au cas de certains aciers [17].

En utilisant le formalisme du critère RDG, on calcule une pression de cavitation de $84 \mathrm{kPa}$ à partir de la valeur de $0,18 \mathrm{~s}^{-1}$ pour la vitesse de déformation viscoplastique. Cet ordre de grandeur est conforme à ce que l'on peut observer pour un alliage d'aluminium $[6,9]$.

\subsection{Conditions de propagation d'une fissure}

Pour la caractérisation des conditions de propagation d'une fissure, des calculs similaires sont réalisés mais en appliquant un relâchement des nœuds à l'arrière du bain, le long de l'axe de la ligne de fusion pour simuler la présence de la fissure. De cette façon, les deux côtés, de part et d'autre de l'axe de symétrie, sont libres de se rapprocher (simulant la guérison de la fissure) ou au contraire de s'éloigner et donc propager la fissure. Sur la figure 8, est présenté le champ de température, dans l'éprouvette fissurée de largeur $30 \mathrm{~mm}$, à trois instants du soudage sur la longueur de l'éprouvette. Ce champ est projeté sur le maillage simulant la propagation de la fissure.

On constate effectivement que la fissure a tendance à s'ouvrir au fur et à mesure de l'avancée du soudage (amplification des déplacements par un facteur 5). En outre, la valeur des deux indicateurs de déformation et vitesse de déformation viscoplastiques est très faible et proche de 0 , même dans la partie transitoire du début d'éprouvette où les seuils restent bien inférieurs à ceux calculés précédemment (Fig. 7).

Un comportement semblable, bien qu'avec une ouverture inférieure, est observé pour une largeur d'éprouvette de $78 \mathrm{~mm}$. Rappelons qu'expérimentalement il n'y a pas de fissure pour cette configuration. Cela signifie que lorsqu'une fissure est amorcée, elle se propage naturellement jusqu'à ce qu'elle rencontre des conditions thermiques ou thermo-mécaniques susceptibles de la refermer et donc de la stopper. Les conditions thermo-mécaniques d'amorçage d'une fissure sont donc beaucoup plus sévères que les conditions de propagation. Ceci pourrait, par ailleurs, expliquer la dispersion importante des résultats de la littérature pour les matériaux métalliques surtout lorsque la longueur des fissures est considérée comme un indicateur de la sensibilité à la fissuration à chaud.

\section{Conclusions et perspectives}

Les conditions d'amorçage de la fissuration à chaud lors du soudage par faisceau d'électrons d'un alliage $\mathrm{CuCr} 1 \mathrm{Zr}$ sont étudiées par une analyse couplée expériences/calculs numériques. Des essais de soudage instrumentés de type JWRI ont permis d'évaluer expérimentalement un seuil en largeur d'éprouvette de $50 \mathrm{~mm}$; la 


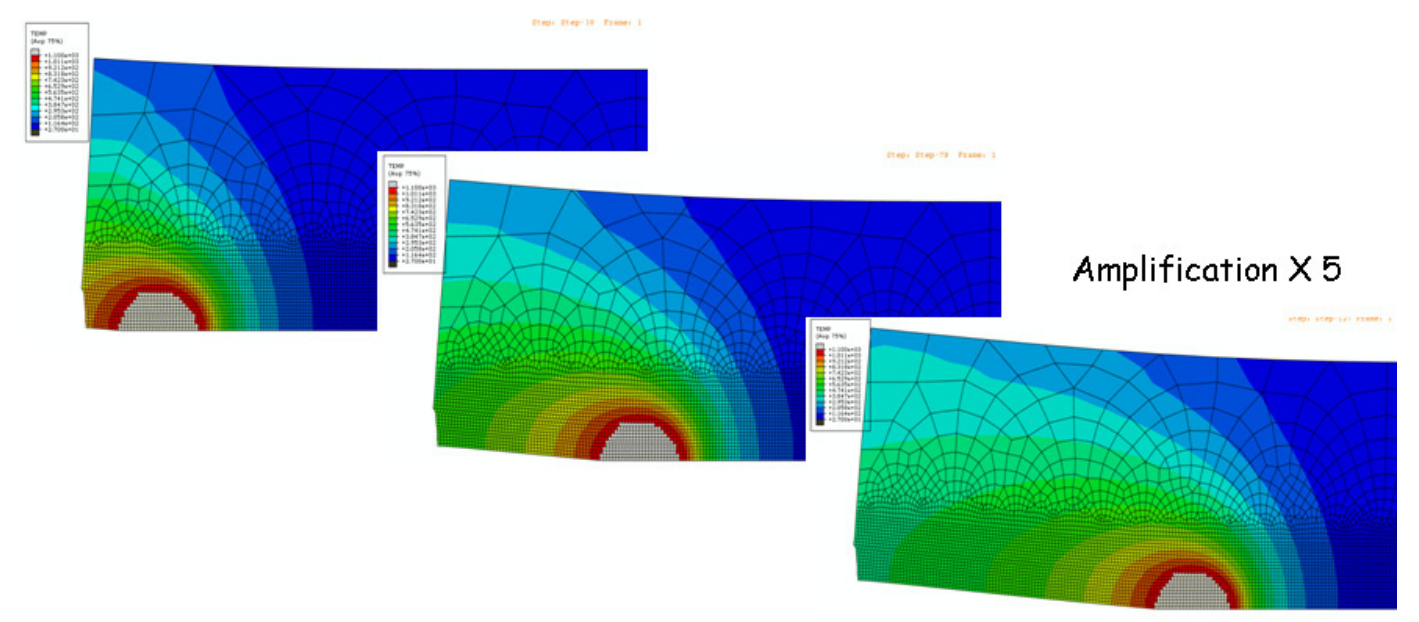

Fig. 8. Simulation de la propagation d'une fissure champ de température à 3 instants du soudage. Fig. 8. Crack propagation simulation. Temperature field at three welding times.

configuration est fissurante pour des éprouvettes de largeur inférieure à cette valeur.

Le calcul des indicateurs issus de deux critères de la littérature, pour cette valeur seuil de largeur d'éprouvette de $50 \mathrm{~mm}$, a permis d'évaluer dans le BTR une déformation transverse viscoplastique de l'ordre de $2,7 \times 10^{-3}$ et une vitesse de déformation transverse viscoplastique proche de $0,18 \mathrm{~s}^{-1}$ correspondant à une pression de cavitation pour cet alliage $\mathrm{CuCr} 1 \mathrm{Zr}$ de $84 \mathrm{kPa}$. On a pu observer par ailleurs que les indicateurs étaient plus importants en début d'éprouvette, en régime transitoire après chute de l'appendice, lieu où s'amorcent effectivement les fissures. Leurs valeurs diminuent ensuite très rapidement dans la partie courante de l'éprouvette.

Enfin, il a été montré que les conditions thermo-mécaniques d'amorçage de la fissure sont beaucoup plus sévères que celles associées à la propagation.

La prochaine étape de l'étude est de vérifier la sensibilité des indicateurs aux conditions opératoires de soudage, notamment à la vitesse et à la puissance pour étudier la transférabilité des critères sur configuration plus complexe. En outre, une meilleure description du comportement mécanique de la zone pâteuse serait nécessaire ainsi qu'une amélioration de la loi de comportement mécanique EVP dans le solide, prenant en compte en particulier les phénomènes de précipitation de l'alliage $\mathrm{CuCr} 1 \mathrm{Zr}$.

\section{Remerciements}

Les auteurs remercient la société «Le Bronze Industriel » qui a soutenu techniquement et financièrement le travail présenté ici ainsi que la région Champagne-Ardenne pour son soutien financier. La machine Gleeble 3500 à l'Université de Bretagne-Sud a été cofinancée par le Fonds Européen de Développement Régional (FEDER).

\section{Références}

[1] M. Lipa, A. Durocher, R. Tivey, Th. Huber, B. Schedler, J. Weigert, Fus. Eng. Des. 75-79 (2005) 469-473

[2] U. Luconi, M. Di Marco, A. Federici, M. Grattarola, G. Gualco, J.M. Larrea, M. Merola, C.Ozzano, G. Pasquale, Fus. Eng. Des. 75-79 (2005) 271-276

[3] A. Durocher, M. Lipa, Ph. Chappuis, J. Schlosser, T. Huber, B. Schedler, J. Nucl. Mat. 307-311 (2002) 1554-1557

[4] M. Rappaz, J.-M. Drezet, M. Gremaud, Metall. Mater. Trans. A 30 (1999) 449-445

[5] J. Campbell, Castings, Elsevier, 2003

[6] J.A. Danzig, M. Rappaz, Solidification, CRC Press, 2009

[7] M. Shibahara, H. Serizawa, H. Murakawa, Math. Mod. Weld Phenomena 5 (2001) 253-267

[8] V. Ploshikhin, A. Prikhodovsky, A. Ilin, C. Heimerdinger, F. Palm, Math. Mod. Weld Phenomena 8 (2007) 87-105

[9] P.T. Houldcroft, Brit. Welding J. (1955) 471475

[10] M. Katoh, K. Nishio, S. Mukae, J. Jap. Welding Soc. 12 (1994) 179-185

[11] F. Matsuda, K. Nakata, Trans. JWRI 12 (1982) 87-94

[12] D.G. Eskin, Suyitno, L. Katgermann, Prog. Mat. Sci. 49 (2004) 629- 711

[13] J.-M. Drezet, M. Rappaz, A New Hot Tearing Criterion for Aluminium Alloys, Proceedings of the 1st Esaform Conference on Material Forming, École des Mines de Paris, 1998

[14] T.W. Clyne, G.J. Davies, Brit. Foundrymen 74 (1981) 65-73

[15] W. Rindler, E. Kozeschnik, B. Buchmayr, Steel Res. 71 (2000) 460-465 
[16] M. Rappaz, A. Jacot, W.J. Boettinger, Met. Mat. Trans. A 34 (2003) 467-479

[17] N. Kerrouault, Fissuration à chaud en soudage d'un acier inoxydable austénitique, Thèse, École Centrale de Paris, 2000

[18] O. Cerri, Rupture à chaud dans les aciers au cours de leur solidification : caractérisation expérimentale et modélisation thermomécanique, Thèse, École des Mines de Paris, 2006

[19] J. Wisniewski, Modélisation thermomécanique de la fissuration à chaud en soudage par faisceau d'électrons d'un alliage $\mathrm{CuCrZr}$, Thèse, Université de Bretagne Sud, 2009

[20] J. Wisniewski, J.M. Drezet, D. Ayrault, B. Cauwe, Temperature, Int. J. Mater. Forming (2008) 1059-1062
[21] J. Wisniewski, D. Carron, M. Carin, J. Costa, D. Ayrault, P. Pilvin, Identification du comportement mécanique d'un alliage $\mathrm{CuCr} 1 \mathrm{Zr}$, Proceedings des Journées annuelles 2009 de la SF2M, Rennes, 2009

[22] P. Pilvin, Notice d'utilisation de SiDoLo, Université de Bretagne-Sud, Version 2.4495, 2003

[23] J.-M. Drezet, D. Ayrault, J. Wisniewski, P. Pilvin, D. Carron, F. Primaux, Experimental and Numerical Analysis of the Hot Tearing Susceptibility of a CuCrZr Alloy, presented at the 9th Int. Seminar on Numerical Analysis of Weldability, Graz, Seggau, 2009

[24] L. Karlsson, Thermal stresses in welding, edited by R.B. Hetnarski, Elsevier, 1986

[25] Y.H. Wei, Z.B. Dong, R.P. Liu, Mod. Simul. Mat. Sci. Eng. 13 (2005) 437-454 